Z Gerontol Geriat 2009 · 42:279-280

DOI 10.1007/s00391-009-0053-5

Online publiziert: 25. Juli 2009

(c) Springer-Verlag 2009

\author{
G. Naegele $\cdot$ M. Sporket \\ Institut für Gerontologie an der Technischen Universität Dortmund
}

\title{
Altern in der Arbeitswelt
}

verstärkten Humankapitalorientierung der Betriebe wird es für die wirtschaftliche Leistungsfähigkeit somit in Zukunft darauf ankommen, nicht nur aktuelles technologisches Know-how von Jüngeren im Austausch mit Älteren in die Unternehmen einzuspeisen, sondern auf einen "gesunden Mix" aus älteren und jüngeren Beschäftigten zu setzen, um das Erfahrungswissen Älterer nutzbar zu machen.

\section{( Ältere Arbeitnehmer werden künftig vermehrt als Arbeitskräfte benötigt}

mehr von derzeit etwa einem knappen Viertel auf mehr als ein Drittel bis 2020 ansteigen wird. Eine von vielen vermuteten Folgen davon ist: Ältere Arbeitnehmer werden künftig wieder vermehrt als Arbeitskräfte benötigt. Für die Unternehmen bedeutet dies, dass die vorzeitige Ausgliederung der älteren Beschäftigten alsbald an ihre Grenzen stoßen könnte und dass womöglich bald wieder ein nennenswerter Bedarf an älteren Arbeitskräften bestehen wird. Eine Lösung, diesen Problemen präventiv zu begegnen, wird darin gesehen, ältere Mitarbeiterinnen und Mitarbeiter länger als bisher im Unternehmen zu halten bzw. zusätzlich auch noch ältere Arbeitsuchende einzustellen. Dies würde allerdings ein Umdenken der Betriebe hinsichtlich ihres bisher praktizierten Externalisierungs- sowie Rekrutierungsprofils voraussetzen.

Schon jetzt hat die jahrzehntelange vorzeitige Externalisierung des Alters aus den Betrieben in einer Reihe von Unternehmen zu einem immensen betrieblichen Wissens- und Erfahrungsverlust geführt, der sich - so wird befürchtet - in einer geminderten gesamtwirtschaftlichen Innovations- und Wettbewerbsfähigkeit niederschlagen könnte [1]. Im Rahmen einer
Hinzu kommt, dass strukturelle Veränderungen in den Lebensläufen der Menschen nicht mehr zu der sich herausgebildeten starren Dreiteilung des Lebenslaufs (verlängerte Ausbildungsphase, verkürzte Erwerbsphase und verlängerte „Ruhestandsphase") passen. Walker [5] spricht z. B. in diesem Zusammenhang von einem „Work-Age-Paradox“, nach dem es keine Begründungen dafür gibt, dass bei positiven Kohorteneffekten, bei Qualifikation und Gesundheit sowie bei ausgeweiteter fernerer Lebenserwartung die Menschen immer früher aus dem Erwerbsleben ausscheiden.

Allerdings lässt sich die Erhöhung der Erwerbsbeteiligung Älterer nicht per se gleichsam durch „Knopfdruck“ realisieren. Es muss vielmehr darauf ankommen, die verschiedenen Förderstrategien für die Beschäftigungsfähigkeit alternder und älterer Arbeitnehmer so anzuwenden, dass im Ergebnis eine Weiterarbeit in der Spätphase des Erwerbslebens nicht nur wünschenswert, sondern auch objektiv möglich ist. Vor allem die betriebliche Ebene sollte dabei im Zentrum der Bemühungen stehen [3], was für Deutschland als durch- gängige Strategie gegenwärtig noch nicht zu erkennen ist. Dazu bedarf es insbesondere längerfristig angelegter Konzepte der Investition in das Humankapital und arbeitsmarkt- und sozialpolitischer Rahmenbedingungen, die diese absichern. Im Kern geht es um eine stärkere Nutzung der ökonomischen Produktivität Älterer in der Arbeitswelt. Der entscheidende Weg dazu ist der von Investitionen in die Arbeits- und Beschäftigungsfähigkeit (,employability") alternder Belegschaften, die vor allem auf betrieblicher Ebene stattfinden müssten. Dazu sind - entsprechend internationaler "Vorbilder" wie Finnland [4] - integrierte, d. h. alle Ebenen der konkreten Arbeits- und Beschäftigungssituation einbeziehende Politikkonzepte vonnöten. Die öffentliche Anerkennung des Themas als ressort- und trägerübergreifende politische Gemeinschaftsaufgabe ist hierzulande jedoch bislang noch nicht "mehrheitsfähig" geworden.

Andererseits besteht bei der politischen Umsetzung dieser Forderung erheblicher Differenzierungsbedarf, denn nicht jeder ältere Beschäftigte ist dazu auch tatsächlich in der Lage. Dies gilt insbesondere für viele ältere Arbeitslose, gesundheitlich Beeinträchtigte, zu denen viele Behinderte zählen, sowie nicht zuletzt für Angehörige besonders belasteter Berufsgruppen und/oder für Arbeitnehmer auf Arbeitsplätzen mit „begrenzter Beschäftigungsdauer", was u. a. durch die immer noch hohen Frühverrentungsquoten wegen Krankheit und/oder vorausgegangener Arbeitslosigkeit bestätigt wird. Die auch real bessere Nutzung des Alters als „Produzent" ökonomischer Leistungen ist und bleibt somit eine in hohem Maß voraussetzungsvolle Aufgabe, die aber - folgt man nationalen und internationalen „Vor- 
bildern“ - zumindest für Teile der älteren Erwerbsbevölkerung von morgen lösbar erscheint.

Die in diesem Schwerpunktthema vorgestellten Beiträge nehmen aus unterschiedlicher Perspektive zu den arbeitsund beschäftigungspolitischen Herausforderungen des demografischen Alterns der Belegschaften Stellung.

Martin Brussig analysiert die Hintergründe der seit einigen Jahren wieder steigenden Erwerbsbeteiligung Älterer und fragt u. a. danach, ob damit bereits eine „Trendwende" eingeläutet worden ist. Er bestätigt in seinem Beitrag eine Zunahme der Beschäftigungschancen Älterer und belegt damit auch, „dass Arbeitsplätze und Leistungsanforderungen auch von vielen Älteren ausgefüllt werden können“. Er bestätigt jedoch auch die Vermutung, dass vor dem Hintergrund der Heterogenität der Gruppe „ältere Arbeitnehmer/ -innen“ die Beschäftigungsaussichten nicht für alle Älteren gleichermaßen günstig sind.

Dies wird auch in dem Beitrag von Verena Leve, Gerhard Naegele und Mirko Sporket deutlich. Die Autoren berichten über eine repräsentative Untersuchung bei älteren Arbeitnehmerinnen aus dem Jahre 2007, in der für die Bundesrepublik bislang erstmals die Beschäftigungssituation von älteren Arbeitnehmerinnen systematisch in den Blick genommen wurde. Anlass der Studie war die im Frühjahr 2007 beschlossene schrittweise Anhebung der Regelaltersgrenze auf 67 (auch für Frauen). Die präsentierten Daten belegen, dass die flächendeckende Realisierung der „Rente mit 67 “ eine hoch voraussetzungsvolle Aufgabe für alle Beteiligten ist und dass dieses Ziel nur von einem Teil der Betroffenen - und dann auch noch unter ganz bestimmten Voraussetzungen erreichbar ist. Ganz wesentlich ist dabei die betriebliche Ebene gefordert.

\section{() Betriebliches Alterns- management kann sich auch betriebswirtschaftlich rechnen}

Mirko Sporket greift diesen Aspekt der betrieblichen Vorleistungsverpflichtungen bei der „Rente mit 67 “ auf und folgt damit der alten These [2], nach der die Umsetzung der Forderung nach Verlängerung der Lebensarbeitszeit vor allem eine betriebliche Aufgabe ist und von daher auch in erster Linie in den Betrieben zu lösen sei. Er konzeptualisiert betriebliches Alternsmanagement und belegt anhand der von ihm untersuchten Fallbeispiele (diese entstammen einer EU-weiten Untersuchung, die im Auftrag der Europäischen Stiftung in Dublin durchgeführt wurde), die positive Wirkung betrieblicher Förderstrategien, -regelungen und -konzepte, wenn es um erfolgreiche Integration Älterer in der Arbeitswelt geht. Es wird dabei ferner deutlich, dass auch die Betriebe von einem produktiven Einsatz älterer Arbeitnehmer profitieren können, dass sich betriebliches Alternsmanagement also auch betriebswirtschaftlich „rechnet“.

Nele Wild-Wall, Patrick Gajewski und Michael Falkenstein beschäftigen sich mit der kognitiven Leistungsfähigkeit älterer Arbeitnehmer. Sie belegen am Beispiel von Beschäftigten eines großen Automobilherstellers einerseits alterstypische Veränderungen von leistungs- und hirnphysiologischen Parametern, belegen andererseits aber auch die These von den Möglichkeiten der Kompensation derartiger Leistungseinbußen, so beispielsweise durch Training und Lernen. Auch sie bestätigen damit anhand ihrer Daten die Notwendigkeit betrieblicher Altersmanagement-Konzepte, wenn sie z. B. auf die kompensatorische Wirkung von Veränderungen an den Arbeitsplätzen (z. B. Rotation) verweisen.

Jürgen Deller und Lena Maxin gehen in ihrem Beitrag auf ein bislang in der gerontologischen Forschung weitgehend unbeachtetes Thema ein: berufliche Aktivitäten im Ruhestand. Ausgehend von dem USamerikanischen Modell des „bridge employment“ wird nach Übertragungsperspektiven und -chancen für Deutschland gefragt. Zugleich liefert der Beitrag einen aktuellen Überblick über Ausmaß und Strukturen beruflicher Aktivitäten in der Nachberufsphase. Darüber hinaus bietet er Hinweise auf Fördermöglichkeiten für eine Politik für „Silver Work“.

Die in diesem Heft veröffentlichten Beiträge zum Thema „Altern in der Arbeitswelt" wurden jeweils in den Monaten März bis Mai 2009 geschrieben. Seither stattgefundene Entwicklungen konnten nicht mehr aufgenommen werden.
Wir bedanken uns ausdrücklich bei den Kolleginnen und Kollegen für ihre rasche Zusage und für ihr unkompliziertes Engagement bei der Mitwirkung an diesem Heft.

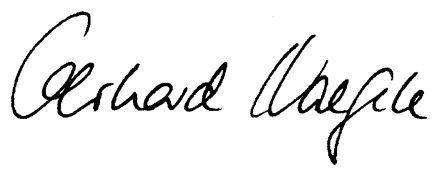

G. Naegele

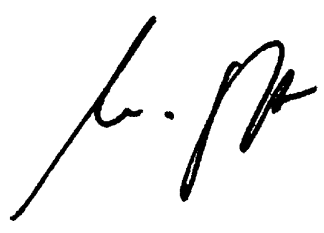

M. Sporket

\section{Korrespondenzadresse \\ Prof. Dr. G. Naegele \\ Institut für Gerontologie \\ an der Technischen Universität Dortmund, Evinger Platz 13, 44339 Dortmund \\ orka@post.uni-dortmund.de}

\section{Literatur}

1. Institut der Deutschen Wirtschaft (2004) Demographischer Wandel. Lust auf Neues. Informationsdienst des Instituts der Deutschen Wirtschaft 24

2. Naegele G (1988) Zur Zukunft älterer Arbeitnehmer - Die Entscheidung für oder gegen die Alterserwerbsarbeit fällt in den Betrieben und ist dort zu beeinflussen. Soziale Sicherheit 6

3. Naegele G, Walker A (2006) A guide to good practice in age management. Office for Official Publications of the European Communities, Luxembourg

4. Vinni K (2002) Active strategies for older workers in Finland. In: Jepsen M, Foden D, Hutsebaut M (eds) Active strategies for older workers. European Trade Union Institute, Brüssel, pp 345-380

5. Walker $A$ (2006) Active ageing in employment: its meaning and potential. Asia-Pacific Review 13:7893 